\title{
Access site pseudoaneurysms after endovascular intervention for peripheral arterial diseases
}

\author{
Ahmed Eleshra ${ }^{1,2}$, Daehwan Kim¹, Hyung Sub Park ${ }^{1,3}$, Taeseung Lee ${ }^{1,3}$ \\ ${ }^{1}$ Department of Surgery, Seoul National University Bundang Hospital, Seongnam, Korea \\ ${ }^{2}$ Department of Vascular Surgery, Faculty of Medicine, Mansoura University Hospital, Mansoura, Egypt \\ ${ }^{3}$ Department of Surgery, Seoul National University College of Medicine, Seoul, Korea
}

\begin{abstract}
Purpose: Pseudoaneurysms after percutaneous vascular access are common and potentially fatal if left untreated. The aim of this study was to determine the incidence and risk factors associated with access site pseudoaneurysms after endovascular intervention for peripheral arterial disease (PAD) under a routine postintervention ultrasound (US) surveillance protocol.

Methods: A total of 254 PAD interventions were performed in a single center between January 2015 and November 2016 , and puncture site duplex US surveillance was routinely performed within 48 hours of the procedure. Clinical, procedural and follow-up US data were analyzed.

Results: The overall incidence of pseudoaneurysm was $2.75 \%$ ( 6 cases in the femoral artery and 1 in the brachial artery). There was no difference between retrograde and antegrade approach, but there was a higher rate of pseudoaneurysm formation after manual compression compared to arterial closure device (ACD) use (4.3\% vs. $0.87 \%$ ). Manual compression was more commonly used for antegrade punctures $(79.0 \%)$ and ACD for retrograde punctures (67.7\%). Calcification was more frequently found in antegrade approach cases (46.8\% vs. $16.9 \%$ for retrograde cases) and manual compression was preferred in its presence. All pseudoaneurysms were treated successfully at the time of diagnosis by US-guided compression repair and there were no cases of rupture.

Conclusion: Pseudoaneurysm rates after therapeutic endovascular intervention for PAD were comparable to other cardiologic or interventional radiologic procedures despite the higher possibility of having a diseased access vessel. Routine US surveillance of access sites allowed for early diagnosis and noninvasive treatment of pseudoaneurysms, preventing potentially fatal complications.

[Ann Surg Treat Res 2019;96(6):305-312]
\end{abstract}

Key Words: False aneurysm, Vascular closure devices, Endovascular procedures, Complications, Peripheral arterial disease

\section{INTRODUCTION}

Percutaneous endovascular revascularization is an established and highly accepted therapy for peripheral arterial diseases (PAD) [1]. It requires deliberate arterial puncture for access, most commonly the common femoral artery, which must be sealed at the end of the procedure. Failure to seal adequately can lead to puncture-related complications, which may include groin hematoma, pseudoaneurysm, arteriovenous fistula, thrombosis, and dissection. Excessive bleeding from the puncture site can be fatal and therefore must not be underestimated, but in some cases of retroperitoneal bleeding,
Received October 5, 2018, Revised March 1, 2019,

Accepted March 28, 2019

Corresponding Author: Taeseung Lee

Department of Surgery, Seoul National University Bundang Hospital, 82

Gumi-ro 173 beon-gil, Bundang-gu, Seongnam 13620, Korea

Tel: +82-31-787-7092, Fax: +82-31-787-4078

E-mail: tslee@snubh.org

ORCID code: https://orcid.org/0000-0001-6425-5924
Copyright (C) 2019, the Korean Surgical Society

(c) Annals of Surgical Treatment and Research is an Open Access Journal. All articles are distributed under the terms of the Creative Commons Attribution NonCommercial License (http://creativecommons.org/licenses/by-nc/4.0/) which permits unrestricted non-commercial use, distribution, and reproduction in any medium, provided the original work is properly cited. 
early diagnosis can be missed. The rate of puncture-related complications has been reported to range from $1.5 \%$ to $9 \%$, and surgical repair is required in $20 \%-40 \%$ of these cases [2,3]. Furthermore, these complications can lead to longer length of hospitalization, increased patient discomfort and an increase in socioeconomic burden. Pseudoaneurysms are one of the troublesome complications that can rupture if left untreated. Some society guidelines have recommended an acceptable rate of $<0.2 \%$, but the reported rates of femoral pseudoaneurysm in the literature vary from as low as $0.2 \%$ to up to $3.8 \%$ of patients when routine duplex ultrasound (US) imaging was performed. $[4,5]$.

Manual compression of the access site is the most widely accepted and cost-effective method of achieving hemostasis after endovascular intervention, but it is claimed to be time consuming and uncomfortable for the patients. Additionally, its mechanism of hemostasis does not lead to direct closure of the vessel, and therefore it is more prone to bleeding complications, especially if patient cooperation is suboptimal. In order to overcome these drawbacks, arterial closure devices have been developed to directly seal the punctured artery. Their efficacy has been proven by many previous studies, and they are nowadays widely used, with the advantage of being comfortable for both the patient and the physician, and allowing for early mobilization and recovery. However, their cost and their indications for use may preclude their routine use in daily practice. Therefore the risks and benefits of arterial closure devices remain a source of controversy [6,7].

The aim of this study was to report the rate of puncturerelated pseudoaneurysm formation after therapeutic endovascular intervention for PAD in a population where routine duplex US surveillance was performed the next day after the intervention. Additionally, the risk factors associated with pseudoaneurysm formation were determined, with special attention to puncture direction and method of hemostasis.

\section{METHODS}

We retrospectively reviewed a prospectively collected data of 273 cases that underwent therapeutic endovascular intervention for occlusive and nonocclusive diseases of the peripheral arterial system from January 2015 to November 2016. The primary aim of this study was to determine the rate of puncture-related pseudoaneurysm formation based on a routine duplex US surveillance protocol the next day after the initial intervention. The patients' clinical characteristics, including medications (antiplatelet and anticoagulation agents), comorbidities and etiology of the disease were analyzed from electronic medical records. Procedural details were also evaluated, which included the site of puncture, direction of access (antegrade vs. retrograde), sheath size, heparin dosage, degree of calcification at the access site (grades 1-4 according to the peripheral arterial calcium scoring system) [8] method of hemostasis, and type of arterial closure device used. We excluded 19 cases that had not undergone follow-up duplex US after the intervention. Additionally, groin hematomas were not included in this study due to the benign nature of progression. The study was approved by the Institutional Review Board of Seoul National University Bundang Hospital (IRB No. B-1801-442105), and informed consent was waived due to the retrospective nature of the study.

\section{Procedure}

All endovascular interventions were performed in a hybrid operation room equipped with a bi-planar fixed C-arm. All procedures were therapeutic in nature and diagnostic cases were not included in this study. Access was obtained using the Seldinger technique under ultrasound-guidance and a micropuncture set with a 21-gauge needle was used routinely for all cases. Areas of calcification were avoided during puncture whenever possible. The direction of access was retrograde for iliac and aortic pathologies or crossover interventions, while antegrade approach was used for femoro-popliteal or below-theknee interventions. Patients were anticoagulated routinely with unfractionated heparin with dosage based on body weight and endovascular procedures were performed by an experienced vascular interventionist with good expertise in endovascular intervention.

\section{Access site management after intervention}

Arterial sheaths were routinely removed in the operation room and hemostasis was achieved either by manual compression or by use of arterial closure devices. The method of hemostasis was mainly decided on the basis of physician preference, but manual compression was preferred for small caliber sheaths or for heavily calcified access sites. Manual compression was performed by the first assistant for at least 20-30 minutes, a compression bandage was then applied, and patients were advised to stay immobilized for at least $6-8$ hours after the procedure. For ACDs, the puncture sites and gloves were sterilized with chlorhexidine solution before application of the devices, and arterial closure was performed according to the instructions for use of each device. The ACDs used were the Perclose ProGlide (Abbott Vascular Inc., Santa Clara, CA, USA), FemoSeal (St. Jude Medical, St. Paul, MN, USA), StarClose (Abbott) and Mynx (Cardinal Health, Dublin, OH, USA). In these patients, bed rest for 4 hours after closure was recommended.

\section{Duplex ultrasound examination}

Duplex US was performed routinely the next day after endovascular intervention by registered vascular technicians in a noninvasive vascular lab. For the femoral artery, the US 
was scanned from the distal external iliac artery down to the proximal portion of the superficial femoral artery, with special attention to the femoral bifurcation. A pseudoaneurysm was defined as a swirling color flow seen in a mass separate from the femoral artery, with color flow within a tract leading from the artery to the mass (consistent with a pseudoaneurysm neck), and a characteristic "to and fro" Doppler waveform in the pseudoaneurysm neck. The maximum diameter of each pseudoaneurysm was determined and documented on transverse and longitudinal views. The shape and diameter of the neck were also measured.

\section{Ultrasound-guided compression repair}

The initial treatment strategy after pseudoaneurysm detection was ultrasound-guided compression repair (UGCR), which was performed using a technique previously described [9]. At the time of detection, gradual pressure was applied under ultrasound guidance with a linear probe to interrupt flow in the pseudoaneurysm neck while allowing flow through the supplying artery. After approximately 10 to 15 minutes of compression, both flow and peripheral pulses were reassessed, and the procedure was repeated at most 3 times (maximum 45 minutes of compression time) if the false lumen and neck were still patent. The patient was kept supine for 3 to 4 hours with the affected leg in a stretched position. A successful UGCR was defined as one that achieved complete thrombosis of the pseudoaneurysm lumen with elimination of flow within the sac. Follow-up US was obtained within 24 hours to detect for any recurrence.

\section{Statistical analysis}

All continuous data are presented as mean \pm standard deviation and categorical data as frequencies and percentages. Statistical analysis for comparison of groups was done using the t-test for continuous variables and chi-squared test for categorical variables. A P-value of less than 0.05 was considered to be statistically significant. All analyses were done using IBM SPSS Statistics ver. 20.0 (IBM Co., Armonk, NY, USA).

\section{RESULTS}

A total of 254 cases (199 patients) with duplex US surveillance after endovascular intervention were analyzed (Fig. 1). The mean age was $73 \pm 12$ years and $85 \%$ were males. The characteristics of the patients are shown in Table 1. The indications for treatment were peripheral arterial occlusive diseases in $75 \%$ of cases, aneurysmal disease including aortic aneurysms in $21 \%$ and acute arterial thrombosis in $4 \%$ of cases. Most accesses were performed via the femoral artery with selected cases of brachial artery access $(n=7)$ when indicated. There were 124 cases of antegrade approach and 130 cases of retrograde approach.

For antegrade approach cases, manual compression was more commonly used ( $79.0 \%$ vs. $21 \%$ for ACD) while for retrograde approach, ACD use was more common (67.7\% vs. $32.3 \%$ for MC). Analyses of parameters related to antegrade versus retrograde approach are shown in Table 2, with subanalysis based on method of hemostasis and type of ACD used. Calcification was more prevalent in antegrade approach cases (46.8\%) compared to retrograde cases $(16.9 \%)$, and manual compression was preferred in these cases irrespective of access direction. A large proportion of ACD use in the retrograde approach group was due to aneurysmal disease in the aortoiliac region which was treated by percutaneous endovascular aneurysm repair (EVAR). These were all large bore sheaths that required at least $2 \mathrm{ACDs}$ for each puncture site.

Based on our post-intervention duplex US surveillance, there were 6 cases of femoral artery pseudoaneurysm (2.4\%) and 1 case of brachial artery pseudoaneurysm (0.39\%). The case of brachial artery pseudoaneurysm was found in the retrograde manual compression group. The rate of pseudoaneurysm formation between antegrade and retrograde approach was

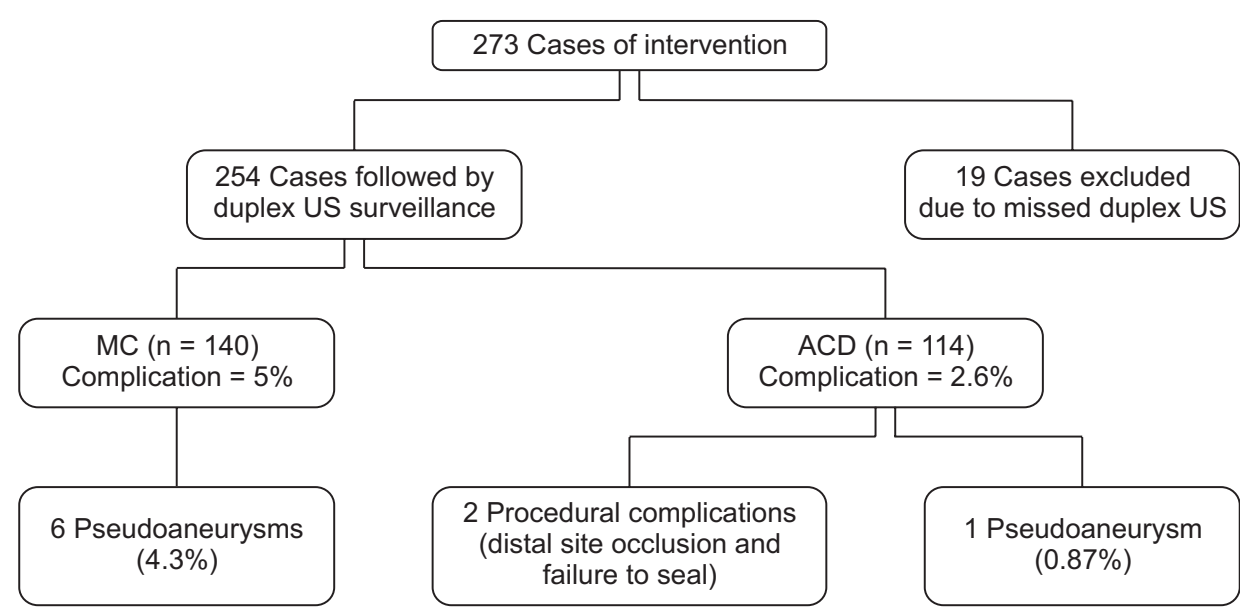

Fig. 1. Overall access site complication rate after therapeutic endovascular intervention during the study period. US, ultrasound; MC, manual compression; ACD, arterial closure device. 
Table 1. Demographic findings of patients with or without pseudoaneurysm

\begin{tabular}{|c|c|c|c|c|}
\hline Variable & $\begin{array}{c}\text { Total } \\
(\mathrm{n}=199)\end{array}$ & $\begin{array}{l}\text { Pseudoaneurysm } \\
\qquad(\mathrm{n}=7)\end{array}$ & $\begin{array}{l}\text { No pseudoaneurysm } \\
(\mathrm{n}=192)\end{array}$ & P-value \\
\hline Age (yr) & $73 \pm 12$ & $70 \pm 14$ & $72.7 \pm 12$ & 0.767 \\
\hline Male sex & $169(85)$ & $7(100)$ & $162(84)$ & 0.501 \\
\hline Body mass index $\left(\mathrm{kg} / \mathrm{m}^{2}\right)$ & $20 \pm 5$ & $19.5 \pm 4$ & $20 \pm 5$ & 0.599 \\
\hline \multicolumn{5}{|l|}{ Comorbidities } \\
\hline HTN & $133(67)$ & $3(42.9)$ & $130(67.7)$ & 0.333 \\
\hline DM & $95(48)$ & $5(71.4)$ & $90(46.9)$ & 0.541 \\
\hline $\mathrm{CKD}^{\mathrm{a})}$ & $36(18)$ & $4(57.1)$ & $32(16.1)$ & 0.413 \\
\hline Ischemic heart disease/stroke & $45(23)$ & $5(71.4)$ & $40(20.8)$ & 0.261 \\
\hline Current smoker & $75(38)$ & $3(42.9)$ & $72(37.5)$ & 0.563 \\
\hline Dyslipidemia & $60(30)$ & $1(14.3)$ & $59(30.7)$ & 0.140 \\
\hline \multicolumn{5}{|l|}{ Indications ${ }^{\mathrm{b})}$} \\
\hline Chronic PAOD & $190(75)$ & $6(85.7)$ & $184(74.5)$ & 0.814 \\
\hline Aneurysm ${ }^{c)}$ & $53(21)$ & $1(14.3)$ & $52(21.1)$ & 0.652 \\
\hline Arterial thrombosis & $11(4)$ & $0(0)$ & $11(4.5)$ & 0.175 \\
\hline
\end{tabular}

Values are presented as mean \pm standard deviation or number $(\%)$.

HTN, hypertension; DM, diabetes mellitus; CKD, chronic kidney disease; PAOD, peripheral arterial occlusive disease.

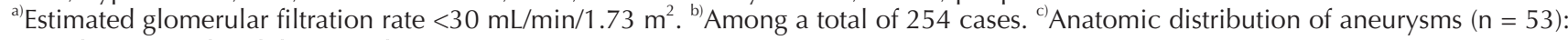
aortoiliac 49, isolated iliac 3, splenic 1.

Table 2. Comparison of access direction according to method of hemostasis

\begin{tabular}{|c|c|c|c|c|}
\hline \multirow{2}{*}{ Variable } & \multicolumn{2}{|c|}{ Antegrade $(\mathrm{n}=124)$} & \multicolumn{2}{|c|}{ Retrograde $(\mathrm{n}=130)^{\mathrm{a})}$} \\
\hline & $\mathrm{MC}(\mathrm{n}=98)$ & $\operatorname{ACD}(n=26)$ & $\mathrm{MC}(\mathrm{n}=42)$ & $\operatorname{ACD}(n=88)$ \\
\hline Calcification $^{\text {b) }}, \mathrm{n}(\%)$ & $57(58.2)$ & $1(3.8)$ & $13(31.0)$ & $9(10.2)$ \\
\hline \multicolumn{5}{|l|}{ Indication } \\
\hline Chronic PAOD & 87 & 26 & 42 & 35 \\
\hline Aneurysm & 0 & 0 & 0 & 53 \\
\hline Arterial thrombosis & 11 & 0 & 0 & 0 \\
\hline \multicolumn{5}{|l|}{ Complication } \\
\hline Pseudoaneurysm, n (\%) & $3(3.1)$ & 0 & $3(7.1)^{c)}$ & $1(1.1)$ \\
\hline Failed hemostasis & 0 & 0 & 0 & 1 \\
\hline Access site occlusion & 0 & 0 & 0 & 1 \\
\hline \multicolumn{5}{|l|}{ ACD type } \\
\hline Perclose ProGlide & - & 0 & - & 49 \\
\hline FemoSeal & - & 4 & - & 39 \\
\hline StarClose & - & 21 & - & 0 \\
\hline Mynx & - & 1 & - & 0 \\
\hline
\end{tabular}

MC, manual compression; ACD, arterial closure device; PAOD, peripheral arterial occlusive disease.

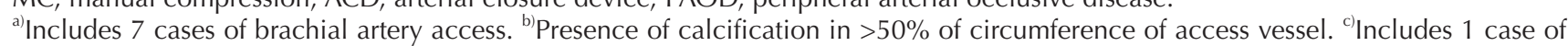
brachial artery access.

similar ( $3.1 \%$ in both groups) but there was a higher predilection for pseudoaneurysm formation after MC in both groups $(3.1 \%$ in antegrade and $7.1 \%$ in retrograde approach, overall $4.3 \%$ ). There was only one case $(0.87 \%)$ of pseudoaneurysm formation after ACD use, which occurred after use of FemoSeal in a retrograde approach case. Other complications included 1 case of failed hemostasis and 1 access site occlusion due to Perclose ProGlide failure during retrograde approach for EVAR which required open conversion. Additionally there were 15 patients with subcutaneous hematoma (5.9\%), but no other local complications (such as arteriovenous fistulas or dissections) were found during duplex US.

The periprocedural characteristics of the cases of pseudoaneurysm formation are shown in Table 3. All pseudoaneurysms occurred after $6 \mathrm{~F}$ sheath use and around $43 \%$ of patients were taking dual antiplatelet/anticoagulation agents preoperatively. A low platelet count of less than $200 \times 10^{9} / \mathrm{L}$ was also found in $43 \%$ of these patients. 
Table 3. Periprocedural findings of cases with or without pseudoaneurysm

\begin{tabular}{|c|c|c|c|}
\hline Variable & $\begin{array}{l}\text { Pseudoaneurysm cases } \\
\qquad(\mathrm{n}=7)\end{array}$ & $\begin{array}{l}\text { No pseudoaneurysm cases } \\
\qquad(\mathrm{n}=247)\end{array}$ & P-value \\
\hline Procedure time (min) & $111 \pm 45$ & $134 \pm 28$ & 0.435 \\
\hline \multicolumn{4}{|l|}{ Access site } \\
\hline Femoral retrograde & $3(42.9)$ & $120(48.6)$ & 0.476 \\
\hline Femoral antegrade & $3(42.9)$ & $121(49.0)$ & 0.463 \\
\hline Brachial retrograde & $1(14.2)$ & $6(2.4)$ & 0.273 \\
\hline \multicolumn{4}{|l|}{ Medications } \\
\hline Double antiplatelet (preoperative) & $2(28.6)$ & $40(16.2)$ & 0.683 \\
\hline Warfarin (preoperative) & $1(14.2)$ & $19(7.7)$ & 0.492 \\
\hline Low platelet count $\left(<200 \times 10^{3} / \mu \mathrm{L}\right)$ & $3(42.9)$ & $24(9.7)$ & 0.074 \\
\hline Calcification $^{\mathrm{a})}$ & $1(14.2)$ & $60(24.3)$ & 0.427 \\
\hline Infection & $0(0)$ & $0(0)$ & - \\
\hline Overlying skin necrosis & $0(0)$ & $0(0)$ & - \\
\hline Deep vein thrombosis & $0(0)$ & $0(0)$ & - \\
\hline
\end{tabular}

Values are presented as mean \pm standard deviation or number (\%).

${ }^{a}$ Presence of calcification in $>50 \%$ of circumference of access vessel.
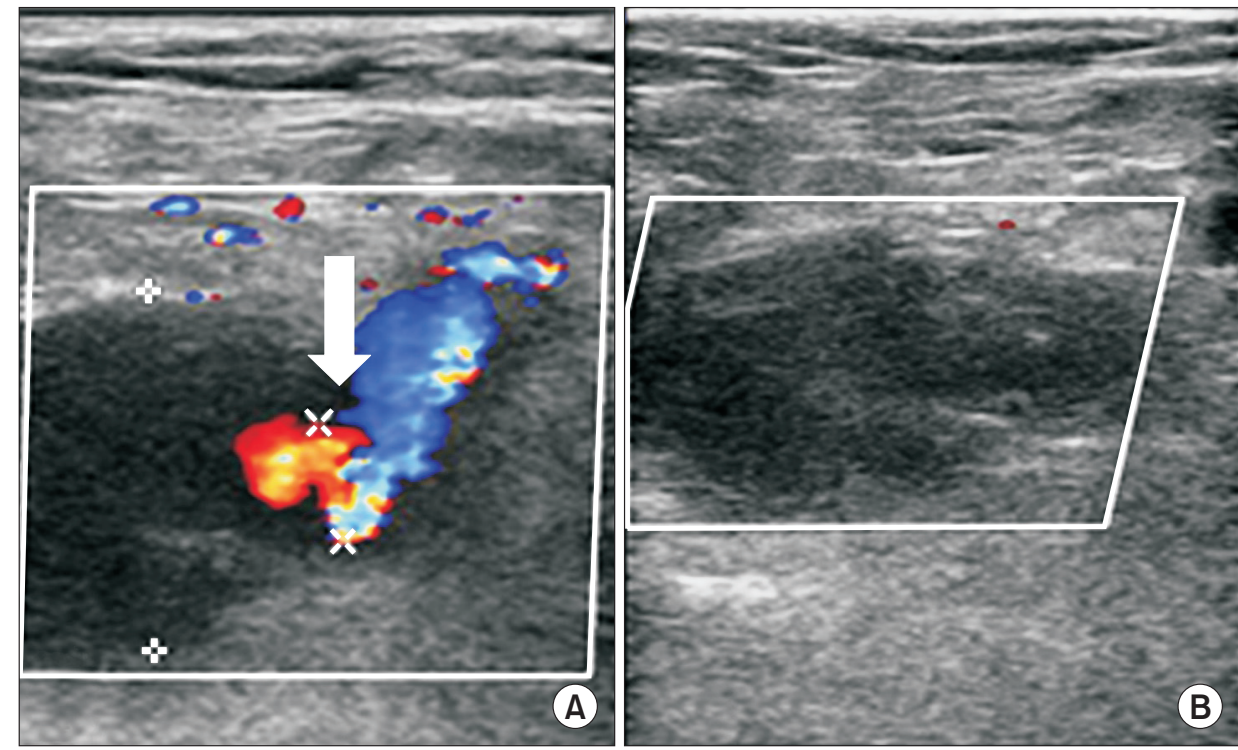

Fig. 2. Duplex ultrasound before and after ultrasound guided compression repair. (A) Before compression with white arrow indicating flow through the neck of the pseudoaneurysm. (B) After compression repair.

Data obtained from duplex US scan regarding pseudoaneurysm characteristics showed that the arterial wall at the puncture site was healthy in 6 patients, while calcification and atherosclerosis was detected in only 1 patient. All pseudoaneurysms were single in nature and the shape ranged from oval or saccular to irregular. The mean diameter of the pseudoaneurysms was $34.0 \mathrm{~mm} \times 16.1 \mathrm{~mm}$ (longitudinal and transverse diameters) and the mean diameter of the pseudoaneurysm neck was $1.4 \mathrm{~mm}$. Doppler waveforms in the pedal arteries were triphasic in all examined patients.

UGCR performed at the time of US diagnosis achieved successful thrombosis of the pseudoaneurysm sac and neck in all patients (Fig. 2). All patients tolerated the UGCR procedure well. Follow-up duplex US after 24 hours showed a well thrombosed sac and all patients were found to be asymptomatic with no evidence of recurrence during clinical follow-up after 30 days.

\section{DISCUSSION}

The overall rate of pseudoaneurysm formation was $2.75 \%$ in our single center study of 254 cases of therapeutic endovascular intervention for PAD during a period of less than 2 years. This overall rate is similar to, or lower than other reports that have reported pseudoaneurysm rates of up to $9 \%$. Although this is a single center study with a relatively small number of patients analyzed, this study describes a real world experience of consecutive patients having undergone routine duplex US surveillance after intervention to screen for the presence of 
pseudoaneurysms. It is important to note that this study only involved cases of therapeutic intervention, which usually requires higher caliber sheaths, more manipulation (due to more frequent device exchange) and longer procedural times than diagnostic procedures, all of which have been reported to be risk factors for pseudoaneurysm formation $[10,11]$. This is the reason why reported rates of pseudoaneurysm formation after diagnostic procedures are around $0.7 \%$ but increases to $3.5 \%-7 \%$ for therapeutic procedures [4]. Furthermore, this study involved cases of EVAR in $21 \%$ of the cases, which require sheaths of up to 22 French, and can be considered to be more prone to access site complications including pseudoaneurysms. Despite the use of higher caliber sheaths and routine use of duplex US for detection of pseudoaneurysms (which can lead to higher reporting rates compared to other studies), the rate of pseudoaneurysm formation in our study was comparable to other major studies.

This study includes a large proportion of antegrade femoral access cases, which is solely performed for infrainguinal lower extremity arterial diseases. Most studies evaluating access site complications or method of hemostasis have been performed for cardiologic or interventional radiologic procedures, which require retrograde femoral approach $[12,13]$. Therefore direct comparison between our study and other major studies may not be suitable. It should be noted that the presence of PAD has been associated with a higher incidence of pseudoaneurysm formation [14]. Calcification is a wellknown factor related to access site complications [15], and our results showed a higher prevalence of calcification in antegrade approach cases. An explanation for this is that many of our cases included treatment of diabetic foot, which is known to be related to medial calcinosis, and also predominantly affect the infrapopliteal vessels, requiring an antegrade approach. Additionally, our retrograde approach cases included cases of aortic aneurysm repair, which may be less prone to calcification, leading to the lower presence of calcification in our retrograde cases.

An analysis of parameters related to pseudoaneurysm formation showed that none of the risk factors analyzed were statistically significant, although the small number of cases with pseudoaneurysm compared to cases without pseudoaneurysm does not allow for a direct comparison between the 2 groups due to lack of statistical power. However, there was a tendency for higher prevalence of comorbidities (especially DM) in this population. Additionally, a platelet count of less than $200,000 / \mu \mathrm{L}$ was also found in around half of the patients. Preoperative low platelet count has been reported to be a risk factor for pseudoaneurysm formation, as well as the use of antiplatelets and/or anticoagulants in both pre- and postprocedural periods [16,17]. Other reported risk factors for pseudoaneurysm formation include advanced age, female gender, increased BMI, larger sheath size and emergency procedures, but our results did not show such significance due to the small numbers.

Our protocol of routine duplex US surveillance after intervention has the advantage of detecting pseudoaneurysms in the early postintervention period, allowing for early treatment. Whether the routine use of duplex US for screening is costeffective is another issue, but it allows for detection of pseudoaneurysms that can go unnoticed by simple physical examination and present later with rupture. As expected, there were no cases of rupture in our patients and all of them were effectively treated by UGCR. UGCR is known to have a success rate of $75 \%-90 \%$ [18-20], but in our study all pseudoaneurysm cases were successfully treated by UGCR without the need for additional, more invasive procedures. Our high success rate of UGCR may be attributed to the fact that pseudoaneurysms were detected at an early phase when the size was relatively smaller and there was no infiltration or inflammation of the tissue surrounding it, allowing for easier and more effective compression. In addition, all of our cases of pseudoaneurysm had a narrow neck, which is known to be a favorable anatomic characteristic for successful thrombosis after UGCR. Although UGCR has the disadvantage of being uncomfortable for both the patient and the operator, it is one of the least invasive ways of treating these pseudoaneurysms.

A subanalysis based on method of hemostasis was also performed, even though this was not a head-to-head comparison between MC versus ACD. Since the decision of whether to use $\mathrm{MC}$ or $\mathrm{ACD}$ was based on physician preference, our study results show that MC was preferred for cases with calcification at the access site. Such strategy is also widely used by many interventionists since it is well known that ACD failure occurs more frequently on calcified access vessels. Based on our comparison, pseudoaneurysms were more common after MC compared to ACD. The main reason may be due to the more calcified or diseased nature of the access vessels in the MC group, which may also correlate with the high proportion of DM in patients with pseudoaneurysm, as previously mentioned ( $71 \%$ vs. $48 \%$ in the overall study population). However another possibility is that $\mathrm{MC}$ is also related to interpersonal differences depending on the expertise of the person who performs the compression (the first assistant in our study). Ates et al. [21] reported that the number of cases in a particular room was an independent risk factor for pseudoaneurysm formation and attributed this to personal factors associated with the person performing the manual compression (including total compression time). Additionally, for MC we routinely performed 30 minutes of compression regardless of sheath size and decided to stop if there was no visual evidence of extravasation, but there are suggestions that compression time should differ based on the size of the sheath used. Therefore 
our uniform protocol of compression may have led to a higher rate of pseudoaneurysm in the MC group. Although ACD use led to lower rates of pseudoaneurysm formation in our study, it must be noted that ACD failure is also very important since it may require open repair (as in 2 cases in our study). Another interesting finding was that we used ACDs in $32 \%$ of our antegrade approach cases, mostly StarClose, but yet there were no cases of pseudoaneurysms. The use of ACDs for antegrade approach is off-label for most devices and data is still lacking, but many recent reports have reported the feasibility and safety of ACD use in antegrade punctures for specific devices [2226], and our experience also advocates its use for antegrade punctures.

Although most of the randomized trials and meta-analyses comparing ACDs versus $\mathrm{MC}$ have failed to show a superiority of ACDs in terms of safety and complication rates, the overall tendency is that ACDs are favored for pseudoaneurysms in particular, since it actively seals the puncture hole limiting the formation of hematoma around the artery subsequently leading to pseudoaneurysm formation. With improvements in ACD technology, and the development of lower profile endovascular devices, one can expect that the rate of pseudoaneurysm formation after ACD use will further decrease.

In conclusion, this study shows that the rate of pseudoaneurysm formation after therapeutic endovascular intervention for PAD is comparable to other cardiologic or radiologic procedures despite the higher possibility of puncturing a diseased access vessel in this specific population. Our routine duplex US screening protocol for puncture site evaluation allowed for early diagnosis and successful noninvasive treatment of this potentially fatal complication. Finally, our study showed that MC was associated with higher rates of pseudoaneurysm formation, probably due to higher rate of calcification, but larger studies with randomization are needed to determine whether ACD is superior to MC.

\section{CONFLICTS OF INTEREST}

No potential conflict of interest relevant to this article was reported.

\section{REFERENCES}

1. European Stroke Organisation, Tendera M, Aboyans V, Bartelink ML, Baumgartner I, Clement D, et al. ESC Guidelines on the diagnosis and treatment of peripheral artery diseases: document covering atherosclerotic disease of extracranial carotid and vertebral, mesenteric, renal, upper and lower extremity arteries: the Task Force on the Diagnosis and Treatment of Peripheral Artery Diseases of the European Society of Cardiology (ESC). Eur Heart J 2011:32:2851-906.

2. Waksman R, King SB 3rd, Douglas JS, Shen Y, Ewing H, Mueller L, et al. Predictors of groin complications after balloon and new-device coronary intervention. Am J Cardiol 1995:75:886-9.

3. Omoigui NA, Califf RM, Pieper K, Keeler G, O'Hanesian MA, Berdan LG, et al. Peripheral vascular complications in the Coronary Angioplasty Versus Excisional Atherectomy Trial (CAVEAT-I). J Am Coll Cardiol 1995:26:922-30.

4. Fransson SG, Nylander E. Vascular injury following cardiac catheterization, coro- nary angiography, and coronary angioplasty. Eur Heart J 1994;15:232-5.

5. Moll R, Habscheid W, Landwehr P. The frequency of false aneurysms of the femoral artery following heart catheterization and PTA (percutaneous transluminal angioplasty). Rofo 1991;154:23-7.

6. Madigan JB, Ratnam LA, Belli AM. Arterial closure devices. A review. J Cardiovasc Surg (Torino) 2007; 48:607-24.

7. Koreny M, Riedmuller E, Nikfardjam M, Siostrzonek P, Müllner M. Arterial puncture closing devices compared with standard manual compression after cardiac catheterization: systematic review and meta-analysis. JAMA 2004:291:350-7.

8. Rocha-Singh KJ, Zeller T, Jaff MR. Peripheral arterial calcification: prevalence, mechanism, detection, and clinical implications. Catheter Cardiovasc Interv 2014;83:E212-20.

9. Coley BD, Roberts AC, Fellmeth BD, Valji K, Bookstein JJ, Hye RJ. Postangiographic femoral artery pseudoaneurysms: further experience with US-guided compression repair. Radiology 1995;194:307-11.

10. Ricci MA, Trevisani GT, Pilcher DB. Vascular complications of cardiac catheterization. Am J Surg 1994;167:375-8.

11. McCleary AJ, Raptis S. Closing the loop: the role of audit in reducing groin complications associated with coronary angiography. Clin Radiol 2001;56:917-21.

12. Nikolsky E, Mehran R, Halkin A, Aymong ED, Mintz GS, Lasic Z, et al. Vascular complications associated with arteriotomy closure devices in patients undergoing percutaneous coronary procedures: a meta-analysis. J Am Coll Cardiol 2004; 44:1200-9.

13. Das R, Ahmed K, Athanasiou T, Morgan RA, Belli AM. Arterial closure devices versus manual compression for femoral haemostasis in interventional radiological procedures: a systematic review and metaanalysis. Cardiovasc Intervent Radiol 2011; 34:723-38.

14. Silber S. Hemostasis success rates and local complications with collagen after femoral access for cardiac catheterization: 
analysis of 6007 published patients. Am Heart J 1998;135:152-6.

15. Stone PA, Campbell JE, AbuRahma AF. Femoral pseudoaneurysms after percutaneous access. J Vasc Surg 2014;60:1359-66.

16. Mlekusch W, Haumer M, Mlekusch I, Dick P, Steiner-Boeker S, Bartok A, et al. Prediction of iatrogenic pseudoaneurysm after percutaneous endovascular procedures. Radiology 2006;240:597-602.

17. Hoke M, Koppensteiner R, Schillinger M, Haumer M, Minar E, Wiesbauer F, et al. D-dimer testing in the diagnosis of transfemoral pseudoaneurysm after percutaneous transluminal procedures. J Vasc Surg 2010:52:383-7.

18. Fellmeth BD, Roberts AC, Bookstein JJ, Freischlag JA, Forsythe JR, Buckner NK, et al. Postangiographic femoral artery injuries: nonsurgical repair with USguided compression. Radiology 1991;178: 671-5.

19. Schaub F, Theiss W, Busch R, Heinz M,
Paschalidis M, Schomig A. Management of 219 consecutive cases of postcatheterization pseudoaneurysm. J Am Coll Cardiol 1997;30:670-5.

20. Khoury M, Rebecca A, Greene K, Rama K, Colaiuta E, Flynn L, et al. Duplex scanning-guided thrombin injection for the treatment of iatrogenic pseudoaneurysms. J Vasc Surg 2002;35:517-21.

21. Ates M, Sahin S, Konuralp C, Gullu U, Cimen S, Kizilay M, et al. Evaluation of risk factors associated with femoral pseudoaneurysms after cardiac catheterization. J Vasc Surg 2006;43:520-4.

22. Gutzeit A, van Schie B, Schoch E, Hergan K, Graf N, Binkert CA. Feasibility and safety of vascular closure devices in an antegrade approach to either the common femoral artery or the superficial femoral artery. Cardiovasc Intervent Radiol 2012; 35:1036-40.

23. Boschewitz JM, Pieper CC, Andersson M, Nadal J, Schild HH, Meyer C. Efficacy and time-to-hemostasis of antegrade femoral access closure using the ExoSeal vascular closure device: a retrospective singlecenter study. Eur J Vasc Endovasc Surg 2014:48:585-91.

24. Chiu AH, Coles SR, Tibballs J, Nadkarni S. The StarClose vascular closure device in antegrade and retrograde punctures: a single-center experience. J Endovasc Ther 2010;17:46-50.

25. Spiliopoulos S, Katsanos K, Karnabatidis D, Diamantopoulos A, Nikolaos C, Siablis D. Safety and efficacy of the StarClose vascular closure device in more than 1000 consecutive peripheral angioplasty procedures. J Endovasc Ther 2011;18:435-43.

26. Looby S, Keeling AN, McErlean A, Given MF, Geoghegan T, Lee MJ. Efficacy and safety of the angioseal vascular closure device post antegrade puncture. Cardiovasc Intervent Radiol 2008;31:558-62. 